\title{
MA Assisted and Restrained Sink Mobility based Economical Data Processing in WSNs: MARS
}

\author{
Shivangi Katiyar ${ }^{1}$, Dr. Rohit Vaid ${ }^{2}$, Dr. Devendra Prasad $^{3}$ \\ ${ }^{I}$ (Research Scholar, Department of Information Technology, MM University, Mullana, Ambala, INDIA) \\ ${ }_{2}^{2}$ (Department of Computer Science and Engineering, MM University, Mullana, Ambala, INDIA) \\ ${ }_{3}^{3}$ (Department of Computer Science and Engineering, Chandigarh Engineering College, Chandigarh, INDIA)
}

\begin{abstract}
Wireless Sensor Network (WSN) is composed of large number of tiny sensor node which have sensing and computation ability. In past WSNs, lots of Data Aggregation scheme has been proposed for prolong network lifetime and eliminate data redundancy. In general sink collects data from all deployed nodes in single processing cycle but region critical WSNs and long communication distance between nodes and sink demands a scheme which process data region wise. The presented scheme adopts sink mobility for region critical data gathering and avails Mobile Agent (MA) for prolonging network lifetime. This paper proposes MA assisted and Restrained Sink mobility based economical data processing in WSNs i.e. MARS. The proposed scheme works for sink trajectory as well as for Data Aggregation. Our simulation result shows that MARS utilizes networks' energy efficiently and prolongs Sensor Network lifetime.
\end{abstract}

Keywords - Mobile sink, Mobile Agent, Data Aggregation

\section{INTRODUCTION}

A sensor network is composed of large number of sensor nodes with limited resources and computation capabilities [1]. According to these computation capabilities it performs sensing and communication related activities. In Wireless Sensor Networks (WSNs) energy is the most delicate factor because nodes have fixed non-rechargeable batteries. There are many issues which are responsible for energy depletion of the node in WSNs and a very common one is transmission of redundant data entries. Redundant data transmission directly indicates non-profitable energy consumption of the nodes. Data Aggregation is the solution for such type of problems which is a process that uses aggregation function for aggregating data from different sources in order to reduce data redundancy during transmission [2]. Long communication distance between sink and sensor nodes drains sensor's energy fast and reduces network lifetime that's why for accelerating network lifetime here we are taking advantage of sink mobility inside the network. Many researchers have been done by considering mobile sink to prolong network lifetime, some of them are discussed in [3-6]. In our proposed method, sink will cover the network area by following prefixed path and after collecting data from one location it will move for another. Sink have prefixed stop points from where it performs data collection. [14-18] Presented scheme introduces controlled mobility of sink and compare it with random mobility of sink for the same network topology. [19-20] The proposed model is Mobile Agent (MA) assisted i.e. For the purpose of data processing, MA travel over the network and generate tree structure path which would be base for every parent node to perform data gathering. The rest of the paper is organized as follows. Section II presents related work. In Section III, we present our system model. Section IV elaborates the Algorithms and Section V Discusses results while Section VI concludes the paper.

\section{RELATED WORK}

In [7] authors consider distance between sink and sensor nodes as a core problem of fast energy depletion of the node during data delivery. That's why they considers mobile sink that will travel the network on fixed path with constant speed. For the purpose of Data Aggregation within the network they worked with Genetic Algorithms. Whereas here genetic algorithm (GA) is used to create energy efficient clusters for data dissemination in wireless sensor networks and performs fitness tests on new structures to select the best population. In a GA, fitness is evaluated by the function defining the problem. The fate of an individual chromosome depends on the fitness value; the better the fitness value, the better the chance of survival. Basic working of the proposed 
method is as; the network is divided into different regions. Each region has one or more sub-sinks based on the number of members in the region. After the regions are splitted a lane is formed and in that lane the mobile sinks moves to collect the data. The member nodes in the region can send the data to mobile sink only via the subsink the sub-sink will transmit the data to mobile sink. This Sub-sink and mobile sink will perform the data aggregation and data is collected using genetic algorithm. Less energy is required to collect the data when genetic algorithm is implemented. Their results are impressive.

In [8] author considers nodes localization and the communication over the network as a main problem and worked for generating an effective communication path so that the effective communication will be performed. In this work, they transmit the data to its nearest node and form a chain to define a reliable and proficient communication over the network. This work includes two main phases, in first nodes localization has been performed and other works for reliable and effective data aggregation. For identifying the aggregative path over the network they considers mobile agent. The path creation process is divided in two main stages, first phase is to identify load of each node and second to create path so that load balancing will be improved. The next hop will be nominated under cost and the reliability criteria.

In [9] authors take advantage of mobile sink for the purpose of designing dynamic routing for static sensor network. They also consider constrained moving of the sink for data gathering and make solution more realistic. They consider with multiple sinks. It broadly examines and evaluates the energy consumption and delay performance for data aggregation and collection with multiple mobile sinks. In order to minimize the maximum energy consumption for $k$ mobile sinks, it proposes optimal scheme to find near optimal $k$ sub-tours with constant estimate. During the stage of data delivery to static sink nodes it gives link scheduling schemes in different cases of transmissions, e.g. with and without aggregation, respectively. The whole scheme works for the objective that is to minimize delivery delay or maximize data rate. Than for data collection with mobile sink it adopts a tour splitting method to get near optimal traveling tours for mobile sinks with constant approximation.

[10], in this paper authors proposed a Mobile Sink Based Reliable and Energy Efficient Data Gathering technique for WSN. Initially they used biased random walk and rendezvous point selection method to find the next position of the sink and the optimal path. It considers that sensor encodes the data by using RS coding and then transmit to the mobile sink. Then the mobile sink decodes the messages and reconstructs the original collection. In proposed method, the pause time of the sink depends on received data and node density. According to their results it improves the performance of the nodes and shows that the proposed technique increases both the reliability and energy efficiency.

In [11], authors consider the presence of multiple static sinks. They start their processing with the generation of sink oriented tree, depending on the requirement of the sink like Interval, Hops, and Timestamps etc. After that data transitions take place. Here the transmission of data is initiated by the nodes which are at a larger distance from the sink, to the following nodes in the tree and the data is transmitted by splitting tree into smaller parts. Data aggregation is performed at nodes nearer to the sink. Their simulation result shows good packet delivery ratio with minimum delay and energy consumption.

In [12] authors propose the SinkTrail protocol that is a novel low-complexity and proactive data reporting protocol for energy efficient data gathering. Here SinkTrail uses logical coordinates to suppose distances and establishes data reporting routes by greedily selecting the shortest path to the destination location. In this method SinkTrail is capable of tracking multiple mobile sinks also. According to author the proposed method successfully eliminates the need of special requirement for special environmental conditions. The simulation results shows that SinkTrail finds shortest data reporting routes and effectively reduces energy consumption.

\section{SYSTEM MODEL}

\section{A. Overview}

This paper proposes a method i.e. mobile sink energy efficient data gathering for WSNs [20-23]. This technique designs a tree structure network and perform data gathering [24-27] with mobile sink, in detail it discussed below. 


\section{B. Network Model}

We consider a $100 \mathrm{~m} * 100 \mathrm{~m}$ area for [28] [29] WSN and the Network area is divided into 4 equal regions. Nodes are deployed by uniform random distribution as shown in Fig.1 (a). Each node can send or receive data packets to its neighbor within a communication range of $34 \mathrm{~m}$. Sink has to move from one region to another using proposed mobility model for data collection. The considered size of MA is 200 bits. The graphical idea behind the proposed scheme is shown in Fig.1 (b).

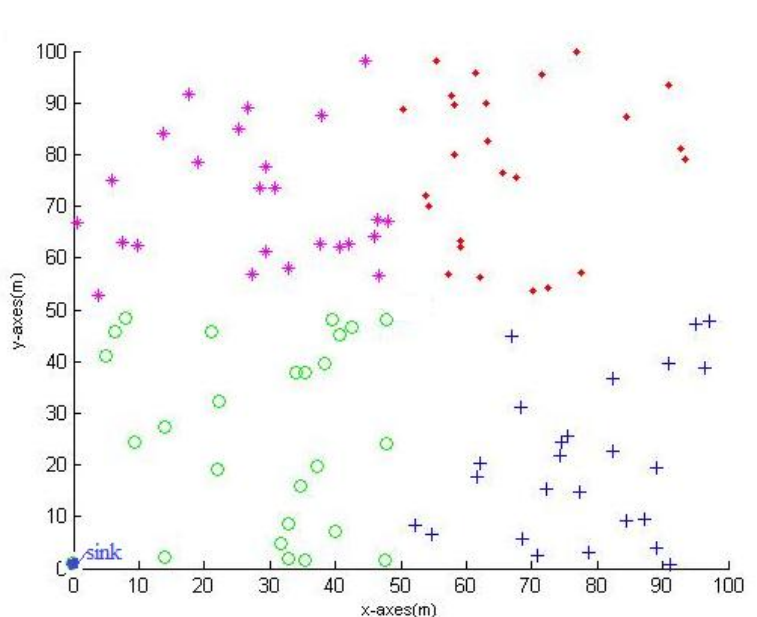

Fig.1 (a) Randomly deployed sensor nodes.

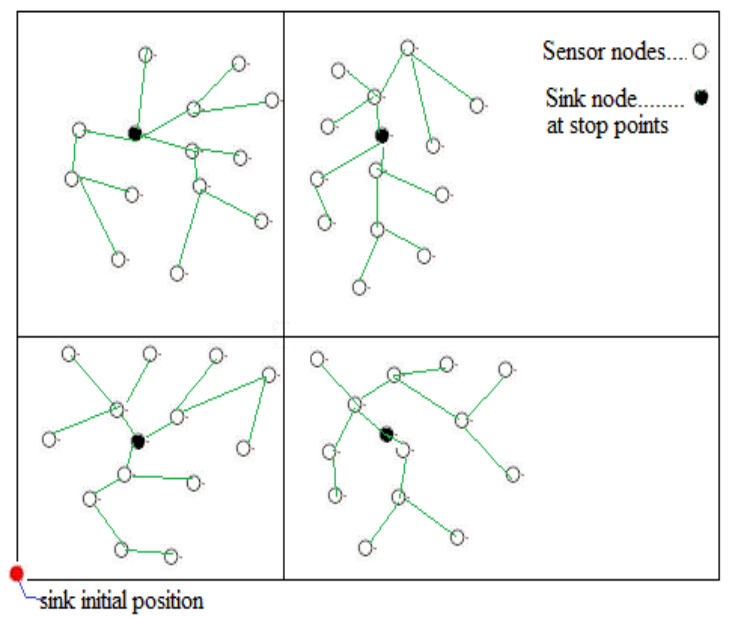

Fig.1 (b) Graphical Idea behind working model

\section{Energy Model}

We employ first order radio model to calculate energy consumption in data transmission by sensors.

$\Theta(\beta, \alpha)=\Theta_{\text {elec }}(\beta)+\Theta-_{\text {amp }}(\beta, \alpha)$

$\Theta_{r x}(\beta)=\Theta_{r x}-{ }_{\text {elec }}(\beta)$

$E D A(\beta)=E D A-{ }_{\text {elec }}(\beta)$

According to first order radio model, $\Theta_{\text {elec }}=50 \mathrm{~nJ} / \mathrm{bit}$ is consumed by the radio to run the transmitter or receiver circuitry and $\Theta_{\text {amp }}=100 \mathrm{pJ} / \mathrm{bit} / \mathrm{m}$ is required for transmitter amplifier, where $\beta$ are number of bits and $\alpha$ is distance. Transmitter circuitry also consumes $E D A=50 \mathrm{~nJ} / \mathrm{bit}$ to aggregate the data received by the child nodes. The sink moves in these regions 1 by 1 , with the particular speed and completes its path in a round. Each sensor compresses the received bits by a data aggregation factor of 0.67 using distributed compressive sampling.

\section{Sink Mobility}

Here scheme considers that sink is rich in any manner either in energy or in computation capabilities and it can vary their Range according to system requirement. It is also assumed that sink is mobile. Proposed method provides a fixed path to sink and it will complete one round when it covers all regions. Each region has a stop point where s ink stops and collect data then moves to next region. In our proposed scheme, the stop point locations will be according to following formula:

$\mathrm{S}_{\mathrm{cp}}=\left\{2 *\left(\mathrm{R}_{\text {length }}\right)\right\}+\mathrm{S}_{\mathrm{ip}}$

Where $\mathrm{S}_{\mathrm{cp}}=$ Current Position of Sink

$\mathrm{S}_{\mathrm{ip}}=$ Initial Position of sink

$\mathrm{R}_{\text {length }}=$ Length of Sub Region;

Whereas $\mathrm{R} 1=\mathrm{R} 2=\mathrm{R} 3=\mathrm{R} 4=\mathrm{R}$. 


\section{ALGORITHMS}

In this section we present Network Formation and Tree Formation Algorithms. Using Algorithm $1 \&$ 2 Mobile Agent travel over the network to build tree structure path to aggregate data. We are adopting Distributed Compressive Sampling (DCS) [13] for data aggregation.

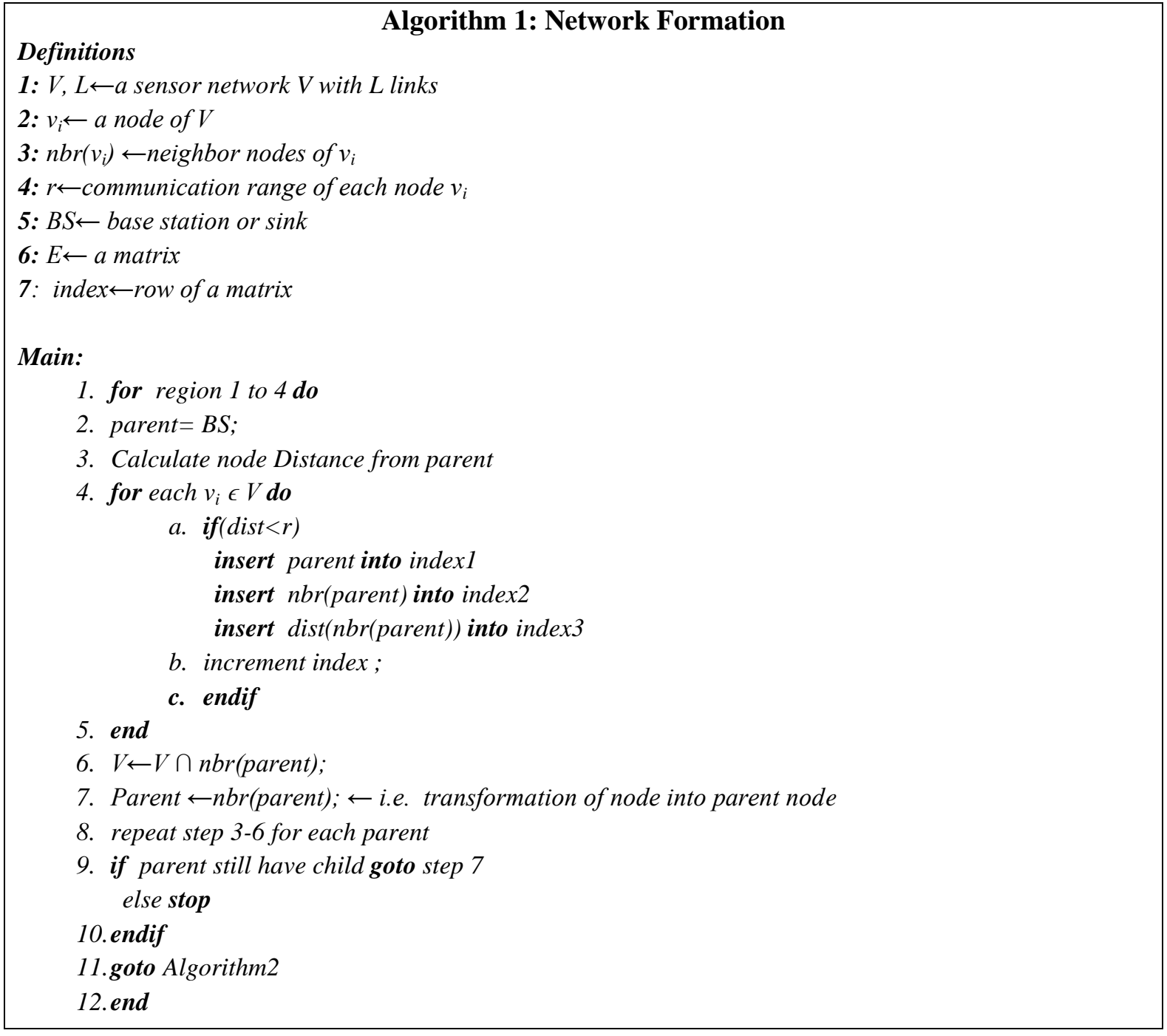

\section{Definitions}

Algorithm 2: Tree Formation

Input $\leftarrow$ Matrix $E$

Main:

Step1: for each index2 of matrix $E$ do

Step2: count no. of node

Step3: if no of parents are greater than 1 compare their distance from node step A:select minimum distance

step B:connect node with minimum distance parent

step4: else connect node with only remaining parent (without comparison).
}

A. Working Steps

The working of proposed scheme is discussed below. 
1. Sink starts to visit all regions of the networks by following equation (4).

2. For each region, take a pause at stop point of region and performs following steps.

a. MA dispatched from sink to travel over the network.

b. MA follows Algorithm1 and Algorithm 2 to generate Aggregation tree.

c. Data gathering starts from leaves of the tree using DCS.

d. Ultimately gathered data will be collected by sink node i.e. root node.

3. Sink switches to the next region.

\section{SIMULATION AND RESULTS}

In this section we analyze the performance of proposed sink mobility based scheme (MARS) which exploits fixed path sink mobility. It is further compared with Random path sink mobility approach (MARS-Random). We performed simulation on MATLAB using simulation parameters shown in Table 1.

Table I

SIMULATION PARAMETERS

\begin{tabular}{|c|c|}
\hline Parameter & Value \\
\hline Network size & $100 \mathrm{~m} * 100 \mathrm{~m}$ \\
\hline Node Number & 100 \\
\hline Packet size & 2000 bits \\
\hline Rounds & 6000 \\
\hline Initial energy of nodes & $0.5 \mathrm{~J}$ \\
\hline Node Range & $34 m$ \\
\hline Initial Sink location & $(\mathbf{0 , 0})$ \\
\hline $\mathbf{E}_{\text {elec }}$ & $50 \mathrm{~nJ} / \mathrm{bit}$ \\
\hline $\mathbf{E}_{f s}$ & $10 \mathrm{pJ} / \mathrm{bit} / \mathrm{m}^{2}$ \\
\hline $\mathbf{E}_{m p}$ & $.0013 \mathrm{pJ} / \mathrm{bit} / \mathrm{m}^{4}$ \\
\hline EDA & 5nJ/bit \\
\hline Size of MA & 200 bits \\
\hline DA (Data Aggregation factor) & 0.67 \\
\hline
\end{tabular}

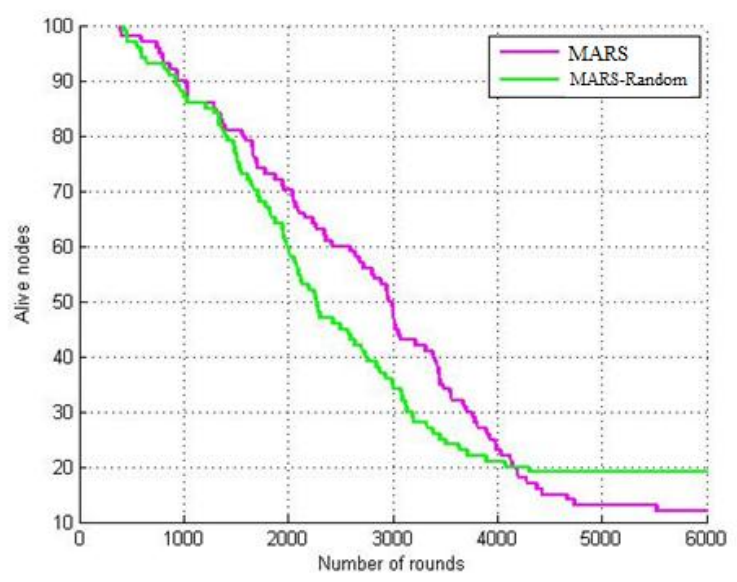

Fig.2: Network Lifetime Graph

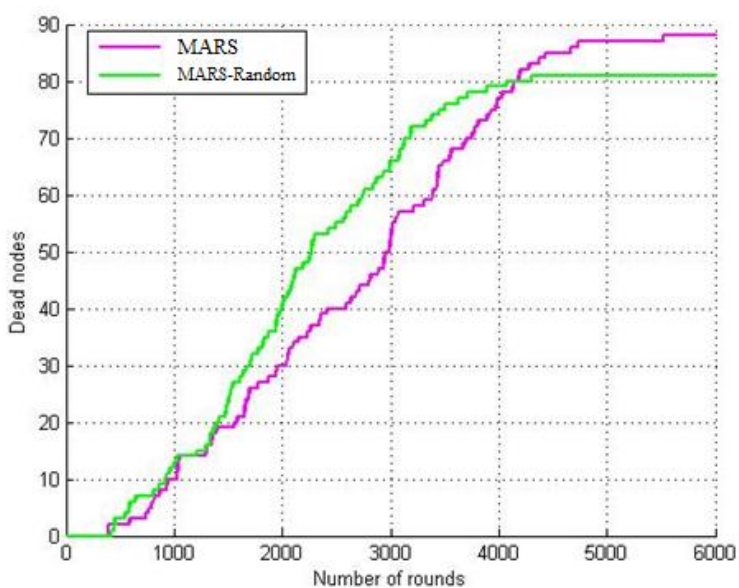

Fig. 3: Comparison of Dead nodes between MARS-Random And MARS 
Fig.2 shows the graph for number of alive nodes during the network lifetime. With the help of figure we can easily see that our method is better than random path sink mobility approach. Remaining alive nodes are unable to communicate with the network because they are located beyond the range of other sensor nodes as well as base station. Also, the residual energy of these sensors is negligibly small and is not sufficient for communication with neighbor nodes. In terms of statistics our proposed method is worked 1200 rounds more than MARS-Random. As we can see all nodes of MARS-Random gets dead approximately at 4300 round but MARS remains still alive after 4300 rounds and crossed 5500 rounds.

Fig. 3 shows the comparison of dead node graph between MARS and MARS-Random. MARS is consuming the energy more efficiently as number of remaining alive nodes is less as compared to MARS-Random. Total number of dead nodes after network disconnection in MARS and MARS-Random is 81 and 88 respectively which represents the efficacy of MARS.

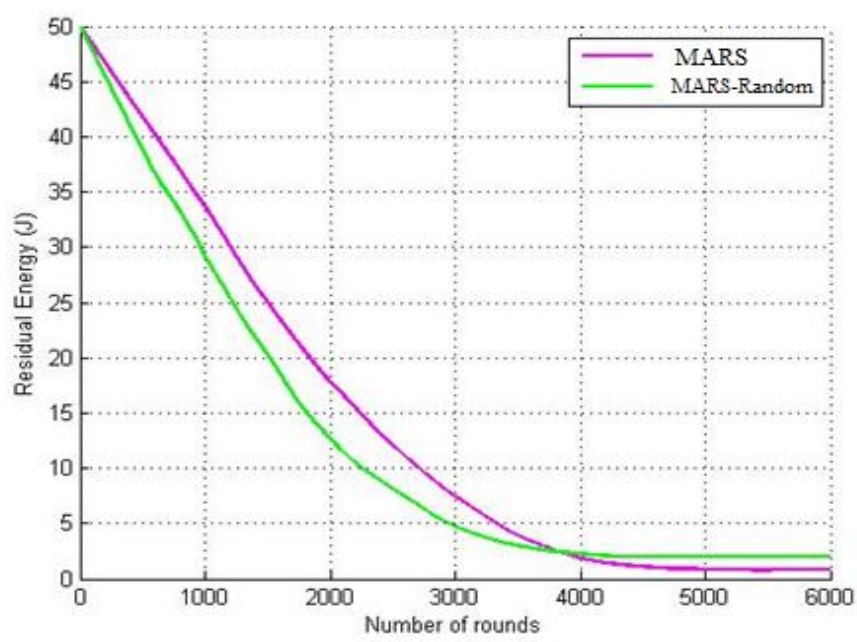

Fig.2: Comparison of Energy consumption in MARS-

Random and MARS

Fig.4 shows the energy consumption of MARS and MARS-Random. Simulation result shows that proposed method consumes energy very efficiently. The MARS-Random exhausted their energy at approximate 4300 rounds but MARS easily crossed 5500 rounds.

\section{CONCLUSION}

MARS prolongs the lifetime of network by dividing the whole network region into four equally spaced regions with controlled sink mobility. Each region of network follows tree level hierarchy for the purpose of data aggregation from sensor nodes to sink. We compared our fixed path sink mobility method MARS with random sink mobility scheme MARS-Random. Results have shown that our method performed the best in terms of network lifetime and efficient utilization of energy. It is also concluded that controlled sink mobility is much effective than random sink mobility

\section{REFERENCES}

[1] Dattatray S. Waghole and Vivek S. Deshpande, "Techniques of Data Collection with Mobile \& Static Sinks in WSN's: A Survey," International Journal of Scientific \& Engineering Research, vol.4, no. 10, pp. 501-505, 2013.

[2] Prof. Deepa.T.P., Ujjwal Jain, Aishwarya N and Nischitha S, "Data Aggregation to improve quality of service in wireless sensor networks: survey", International Journal of Computer Science and Mobile Computing, vol.3, no. 10, pp.783-790, 2014.

[3] J. Luo and J. Hubaux, "Joint mobility and routing for lifetime elongation in wireless sensor networks," IEEE Infocom, vol. 3, 2005. 
[4] M. Younis, M. Bangad, and K. Akkaya, "Base-station repositioning for optimized performance of sensor networks," in VTC 2003.

[5] Y. Shi and Y. Hou, "Theoretical results on base station movement problem for sensor network," in IEEE INFOCOM, 2008, pp. 1-5.

[6] Z. Wang, S. Basagni, E. Melachrinoudis, and C. Petrioli, "Exploiting sink mobility for maximizing sensor networks lifetime," in IEEE HICSS.

[7] K.Praveena and T.Sripriya, "Genetic Algorithm Based Data Aggregation Using Mobile Sink In Wireless Sensor Networks", International Journal of Engineering Research \& Technology (IJERT), Vol.2, Issue 2, February 2013, pp. $1-4$.

[8] Pranavi Tayal, Hardwari Lal Mandoria and Himanshu Chanyal, "Energy Efficient agent based approach for data aggregation in wireless sensor network", International Journal of Wired and Wireless Communications, Vol.2, Issue 1 , October 2012, pp. 30-34.

[9] ShaoJie Tang, Jing Yuan, Xiang Yang Li, Yunhao Liu, GuiHai Chen, Ming Gu and JiZhong Zhao Guojun Dai, "DAWN: Energy Efficient Data Aggregation in WSN with Mobile Sinks", in proceeding of $18^{\text {th }}$ IEEE conference on quality of service (IWQoS), June 2010, pp.1-9.

[10] P. Madhumathy and D. Sivakumar, "Mobile Sink Based Reliable and Energy Efficient Data Gathering Technique For WSNs", Journal of Theoretical and Applied Information, Vol. 61, Issue 1, March 2014, pp. 1-9.

[11] Mohamed Yacoab M.Y. and Dr.V.Sundaram, "Multiple Sink Based Compressive Data Aggregation Technique for Wireless Sensor Network", International Journal of Wireless \& Mobile Networks (IJWMN), Vol. 3, No. 2, April 2011, pp. 182-194.

[12] Xinxin Liu, Han Zhao, Xin Yang, Xiaolin Li and Ning Wang, "Trailing Mobile Sinks: A Proactive Data Reporting Protocol for Wireless Sensor Networks", $7^{\text {th }}$ international conference on Mobile Adhoc and Sensor System (MASS), November 2010, pp. 214-223.

[13] Caione, C., D. Brunelli and L. Benini, "Distributed compressive sampling for lifetime for wireless sensor networks", Industrial Informatics, IEEE, Vol.8,No.1,2012,pp.30-40

[14] Jitender Grover, Shikha Sharma and Mohit Sharma, "Reliable SPIN in Wireless Sensor Network: A Review", IOSR Journal of Computer Engineering (IOSR-JCE), ISSN: 2278-0661, Vol. 16, Issue 6(III), DOI: 10.9790/0661-16637983, pp. 79-83, Nov.-Dec. 2014

[15] Jitender Grover, Shikha Sharma and Mohit Sharma, "A Study of Geographic Adaptive Fidelity Routing Protocol in Wireless Sensor Network", IOSR Journal of Computer Engineering (IOSR-JCE), ISSN: 2278-0661, Volume 16, Issue 5, Ver. IV, DOI: 10.9790/0661-16548896, pp. 88-96, Sep-Oct. 2014. (I.F. 1.686)

[16] Sandeep Singh, Jitender Grover, Randeep Singh Chib, "Review Study of Wireless Sensor Networks, Architecture and Design Issues Related to WSN", Journal of Engineering Research and Studies, Vol. IV, Issue I, Jan.-March, 2014.

[17] Jitender Grover and Shivangi Katiyar, "Agent Based Dynamic Load Balancing in Cloud Computing", IEEE International Conference on Human Computer Interactions (ICHCI'13), Saveetha University, Chennai, DOI: 10.1109/ICHCIIEEE.2013.6887799, pp. 1-6, 23-24, August 2013

[18] J. Polastre, J. Hill, and D. Culler, Versatile low power media access for wireless sensor networks, in The Second ACM Conference on Embedded Networked Sensor Systems (SenSys), pp. 95-107, November 2004.

[19] Jitender Grover \& Reena Rani, "Probabilistic Density Based Adaptive Clustering Scheme to Improve Network Survivability in WSN", IEEE Fifth International Conference on Computing, Communications and Networking Technologies (ICCCNT 2014), Hefei, Anhui, China, DOI: 10.1109/ICCCNT.2014.6963132, pp. 1-7, July 11-13, 2014.

[20] Jitender Grover, Shikha and Mohit Sharma, "Cloud Computing and Its Security Issues - A Review", IEEE Fifth International Conference on Computing, Communications and Networking Technologies (ICCCNT 2014), Hefei, Anhui, China, DOI: 10.1109/ICCCNT.2014.6962991, pp. 1-5, July 11-13, 2014

[21] Jitender Grover, Shikha and Mohit Sharma, "Location Based Protocols in Wireless Sensor Network - A Review", IEEE Fifth International Conference on Computing, Communications and Networking Technologies (ICCCNT 2014), Hefei, Anhui, China, DOI: 10.1109/ICCCNT.2014.6962990, pp. 1-5, July 11-13, 2014.

[22] Jitender Grover, Shikha Sharma and Mohit Sharma, "Optimized GAF in Wireless Sensor Network", IEEE 3rd International Conference on Reliability, Infocom Technologies and Optimization (ICRITO 2014), Amity University, Noida, DOI: 10.1109/ICRITO.2014.7014686, pp. 01-06, October 8-10, 2014.

[23] Jitender Grover, Shikha Sharma and Mohit Sharma, "Reliable SPIN in Wireless Sensor Network", IEEE 3rd International Conference on Reliability, Infocom Technologies and Optimization (ICRITO 2014), Amity University, Noida, DOI: 10.1109/ICRITO.2014.7014694, pp. 01-06, October 8-10, 2014.

[24] A. El-Hoiydi and J. Decotignie, Low power downlink mac protocols for infrastructure wireless sensor networks, ACM Mobile Networks and Applications, vol. 10 (5), pp. 675-690, 2005

[25] Pankaj Pardesi and Jitender Grover, "Improved Multiple Sink Placement Strategy in Wireless Sensor Networks", 2015 IEEE International Conference on Futuristic Trends on Computational Analysis and Knowledge Management (A-BLAZE), Amity University, Greater Noida, Uttar Pradesh, India, DOI: 10.1109/ABLAZE.2015.7155032, pp. 418-424, 25-27 Feb, 2015.

[26] Jitender Grover and Anjali, "Wireless Sensor Network in Railway Signalling System", The IEEE International Conference on Communication Systems and Network Technologies (CSNT-2015), Shri Ram Group of Institutes, Gwalior, DOI10.1109/CSNT.2015.28, pp. 308-313, April 04-06, 2015.

[27] Renu Sharma and Jitender Grover, "Mitigation of Byzantine attack using Enhanced Cooperative Bait Detection and Prevention Scheme (ECBDPS)", 4th IEEE International Conference on Reliability, Infocom Technologies and Optimization (ICRITO-2015), Amity University, Noida, DOI: 10.1109/ICRITO.2015.7359296, pp. 1-6, September 2-4, 2015.

[28] Avinash More, Vijay Raisinghani, Random Backoff Sleep Protocol for Energy Efficient Coverage in Wireless Sensor Networks, Smart innovation,System and Technology,Springer,Varlag, vol. 28 (2), pp. 323-331, 2014.

[29] Michael Buettner, Gary V. Yee, Eric Anderson, and Richard Han, X-MAC: A Short Preamble MAC Protocol for Duty-Cycled Wireless Sensor Networks, in Proceedings of the 4th International Conference on Embedded Networked Sensor System , pp. 307320,2006 